\title{
Predicting antibiotic resistance, not just for quinolones
}

\author{
Seánín M. McCluskey* and Charles W. Knapp \\ Department of Civil Engineering, David Livingstone Centre for Sustainability, University of Strathclyde, Glasgow, UK \\ ${ }^{*}$ Correspondence: seanin.mccluskey@strath.ac.uk
}

\section{A commentary on}

Quinolone resistance: much more than predicted

by Hernández, A., Sánchez, M. B., and Martínez, J. L. (2011). Front. Microbiol. 2:22. doi: 10.3389/fmicb.2011.00022

The ability to better understand and predict the onset of antibiotic resistance to new drugs is needed. Antibiotic resistance has become an emerging issue of concern, and there are emphases to minimize its escalation. The number of cases of antibiotic resistant infections is increasing, as are the numbers of multidrug resistant bacteria. As a result, more antibiotics are becoming of limited use and previous attempts to tackle the resistance problem remain relatively ineffective. If we can more effectively anticipate how and when resistance is likely to arise, we can better manage drug use and perhaps extend efficacy of chemical treatment.

Hernández et al. (2011) discuss how quinolones were introduced as novel antibiotics, and being synthetic, there were no pre-existing resistance mechanisms anticipated. The logic was that if the antibiotics were not synthesized by bacteria (or derived from a bacterially synthesized antibiotic), there would be fewer resistance genes harbored in nature. As such, it was originally anticipated resistance would arise solely from mutations.

There are four types of resistance mechanisms: modification of the antibiotic, modification of the target site, efflux of the drug, and reduced permeability of the cell. Natural antibiotics would be anticipated to have resistance genes falling into some, if not all of these categories. Semi-synthetic antibiotics are merely modified natural antibiotics, so they attack the same targets and have similar molecular structures as their predecessors. Therefore, it would be expected that they would be susceptible to the same resistance mechanisms as those for natural antibiotics. Synthetic drugs, such as quinolones, do not have a similar structure to any pre-existing drugs, so the only type of resistance that they could be susceptible to is modification of the drug site, as it may already be the target of another class of antibiotics.

The same rationale was applied to oxazolidinones, a new class of antibiotics discovered in the 1980s (Livermore, 2003) with the first drug, linezolid, released to market in 2000. The drug's mechanism of action involves the inhibition of the formation of the 70S-ribosome complex, preventing protein synthesis. As bacteria have more than one 23S-rRNA gene, it was thought that resistance arising via mutations would be less likely to occur (Meka and Gold, 2004). As a result of this, linezolid was earmarked as a drug of last resort against methicillin resistant Staphylococcus aureus (MRSA) and vancomycin resistant Enterococci (VRE).

Resistance to linezolid appeared very quickly. By 2001, resistant strains of MRSA and VRE were observed (Taubes, 2008). Linezolid is the only drug in its class approved for use, and it was highly surprising when resistance in the form of the $c f r$ gene appeared (Mendes et al., 2008; Morales et al., 2010). Cfr confers resistance to several drugs - phenicols, lincosamides, oxazolidinones, pleuromutilins, and streptogramin A $\left(\mathrm{PhLOPS}_{\mathrm{A}}\right)$. While these drugs are chemically unrelated, $c f r$ causes methylation of a nucleotide that is in the region of the ribosome these drugs bind (Long et al., 2006). This mutation inhibits the drug-target interaction rendering the antibiotics ineffective.

It could be argued, with many drugs designed to attack the same target, resistance to linezolid should not have been surprising. As previously mentioned, modification of the drug site resistance is not unexpected with synthetic drugs. However, linezolid has a unique interaction with the ribosome, so it was thought it would overcome the resistance issues faced by other drugs classes (Long et al., 2006; Toh et al., 2007). Judging by how quickly resistance appeared, clearly this assumption was incorrect.

When resistance to quinolones appeared, it became apparent that it was caused by more than just the anticipated mutations.
Resistance to quinolones was conferred by drug efflux pumps, target protecting proteins, and quinolone modifying enzymes. In particular, mechanisms for target protection, and drug modification are of particular concern as there is no explanation how these mechanisms arose.

Qnr is a quinolone resistance gene that originated in aquatic bacteria, which made its way into clinical isolates (probably as a result of quinolones being used in fish farming). Environmental bacteria are diverse; despite advances in metagenomics, many bacteria and functional genes have yet to be identified. Sources of resistance may simply be (at this time) unknown. Cfr originated on plasmids in bacterial strains associated with animals [possibly swine (Kehrenberg et al., 2008)] but managed to make it into the chromosome of human bacterial strains (Toh et al., 2007) despite linezolid having never been used agriculturally. Hernández et al. (2011) have already outlined the shortcomings in forecasting quinolone resistance. The inability to predict quinolone inactivating enzymes was based on the fact that there were no known enzyme homologs. Proteins with different primary structures and functions may in fact have similar quaternary structures, so the appearance of deactivating enzymes should not be written off as highly unlikely.

Currently, new oxazolidinones are being developed for eventual clinical use (Prasad, 2007). The examples of both quinolone and linezolid resistance development indicate that better models for the prediction of antibiotic resistance need to be developed, with additional parameters being taken into consideration. Certainly what Hernández et al. (2011) presented is not an isolated case. If we are better able to predict resistance we may be able to extend the shelf life of these new drugs longer than their predecessors.

Resistance to new drugs happens, despite drug novelty. We should consider bacterial diversity, possible exposure to and mobility of genetic traits, and other factor that may exacerbate mutagenesis and gene dissemination, such as environmental pollution. We can only hope that by limiting external 
factors, such as these, the resistance gene reservoir may eventually decline and prolong drug efficacy.

\section{REFERENCES}

Hernández, A., Sánchez, M.B., and Martínez, J. L. (2011). Quinolone resistance: much more than predicted. Front. Microbiol. 2:22. doi: 10.3389/ fmicb.2011.00022

Kehrenberg, C., Cuny, C., Strommenger, B., Schwarz, S., and Witte, W. (2008). Methicillin-resistant and -susceptible Staphylococcus aureus strains of clonal lineages ST398 and ST9 from swine carry the multidrug resistance gene cfr. Antimicrob. Agents Chemother. 53, 779-781.

Livermore, D. M. (2003). Linezolid in vitro: mechanism and antibacterial spectrum. J. Antimicrob. Chemother. 51(Suppl. S2), ii9-ii16.

Long, K. S., Poehlsgaard, J., Kehrenberg, C., Schwarz, S., and Vester, B. (2006). The Cfr rRNA methyltrans- ferase confers resistance to phenicols, lincosamides, oxazolidinones, pleuromutilins, and streptogramin A Antibiotics. Antimicrob. Agents Chemother. 50, 2500-2505.

Meka, V. G., and Gold, H. S. (2004). Antimicrobial resistance to linezolid. Clin. Infect. Dis. 39, 1010-1015.

Mendes, R.E., Deshpande, L.M., Castanheira, M., DiPersio, J., Saubolle, M. A., and Jones, R. N. (2008). First report of cfr-mediated resistance to linezolid in human staphylococcal clinical isolates recovered in the United States. Antimicrob. Agents Chemother. 52, 2244-2246.

Morales, G., Picazo, J. J., Baos, E., Candel, F. J., Arribi, A., Peláez, B., Andrade, R., de la Torre, M. A., Fereres, J., and Sánchez-García, M. (2010). Resistance to linezolid is mediated by the cfr gene in the first report of an outbreak of linezolid-resistant Staphylococcus aureus. Clin. Infect. Dis. 50, 821-825.

Prasad, J. V. (2007). New oxazolidinones. Curr. Opin. Microbiol. 10, 454-460.

Taubes, G. (2008). The bacteria fight back. Science 321, 356-361.
Toh, S. M., Xiong, L., Arias, C. A., Villegas, M. V., Lolans, K., Quinn, J., and Mankin, J. S. (2007). Acquisition of a natural resistance gene renders a clinical strain of methicilin-resistant Staphylococcus aureus resistant to the synthetic antibiotic linezolid. Mol. Microbiol. 64, 1506-1514.

Received: 03 August 2011; accepted: 16 August 2011; published online: 01 September 2011.

Citation: McCluskey SM and Knapp CW (2011) Predicting antibiotic resistance, not just for quinolones. Front. Microbio. 2:178. doi: 10.3389/fmicb.2011.00178

This article was submitted to Frontiers in Antimicrobials, Resistance and Chemotherapy, a specialty of Frontiers in Microbiology.

Copyright ( 92011 McCluskey and Knapp. This is an openaccess article subject to a non-exclusive license between the authors and Frontiers Media SA, which permits use, distribution and reproduction in other forums, provided the original authors and source are credited and other Frontiers conditions are complied with. 